\title{
An optical counterpart to GRB 971227?
}

\author{
A. Guarnieri ${ }^{1}$, A.J. Castro-Tirado ${ }^{2}$, C. Bartolini ${ }^{1}$, M. Lolli ${ }^{1}$, N. Masetti ${ }^{3}$, A. Piccioni ${ }^{1}$, F. Zavatti $^{1}$, J. Gorosabel ${ }^{2}$, \\ Y. Aguilar ${ }^{4}$, R. Kohley ${ }^{4}$, G.M. Beskin ${ }^{5}$, M.R. Zapatero-Osorio ${ }^{6}$, R. Rebolo ${ }^{6}$, R. Corradi $^{6}$, M. Guerrero ${ }^{6}$, S. Kemp ${ }^{6}$,

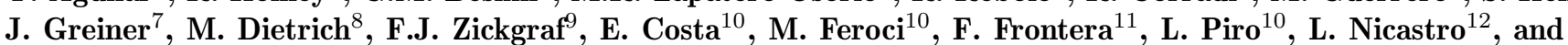 \\ E. Palazzi ${ }^{3}$
}

1 Dipartimento di Astronomia, Università di Bologna, via Zamboni 33, I-40126 Bologna, Italy

2 LAEFF-INTA, Madrid, Spain, and Instituto de Astrofísica de Andalucía (IAA-CSIC), Granada, Spain

3 Istituto Te.S.R.E., CNR, Bologna, Italy

${ }^{4}$ Sternwarte der Universität, Bonn, Germany

${ }_{5}$ Special Astrophysical Observatory, Russia

6 Instituto de Astrofísica de Canarias, La Laguna, Spain

7 Astrophysikalisches Institut, 14482 Potsdam, Germany

8 Landessternwarte Heidelberg, Germany

9 Max-Planck-Institut für Astronomie, Heidelberg, Germany

10 Istituto di Astrofisica Spaziale, CNR, Frascati, Italy

11 Dipartimento di Fisica, Università di Ferrara, Italy

12 IFCAI, CNR, Palermo, Italy

Received December 29, 1998; accepted May 3, 1999

Abstract. Observations taken at Calar Alto and Loiano led to the possible identification of the GRB 971227 optical counterpart. Details on the observations are presented.

Key words: gamma-rays: bursts

\section{Introduction}

Among the GRBs detected by BeppoSAX and Rossi-XTE for which an optical transient (OT) has been proposed, GRB 970228, GRB 980326 and GRB 980519 showed a fast initial fading. The existence of an OT related to GRB 971227 (Castro-Tirado et al. 1997; Bartolini et al. 1997) is less firmly established. If this could be confirmed, there would be growing evidence for a GRB population with short-lived afterglows. Here we present and briefly discuss the results of the optical observations on the error box of the X-ray afterglow of GRB 971227.

\section{The observations}

GRB 971227 was detected by BeppoSAX on December 27.349 UT (Coletta et al. 1997). Two X-ray sources were soon after identified in the GRB error circle (radius of $\left.10^{\prime}\right)$. One of these, 1SAX J1257.3+5924, disappeared approximately 22 hours after the $\gamma$ burst and therefore was proposed as the best candidate for the X-ray afterglow (Piro et al. 1997). Its position $\left(\alpha_{2000}=12^{\mathrm{h}} 57^{\mathrm{m}} 15^{\mathrm{s}}\right.$, $\delta_{2000}=+59^{\circ} 24^{\prime} 02^{\prime \prime}$, error circle radius of 1.5 ) was at the northern boundary of the error box of GRB 971227 .

Among the images of the GRB 971227 field taken by Castro-Tirado et al. (1997) at the 2.2-m German-Spanish telescope at Calar Alto, only one on December 27.90 UT (about 13 hours after the $\gamma$ flare) included the entire error

$\overline{\text { Send offprint requests to: }}$ adriano@astbo3.bo.astro.it

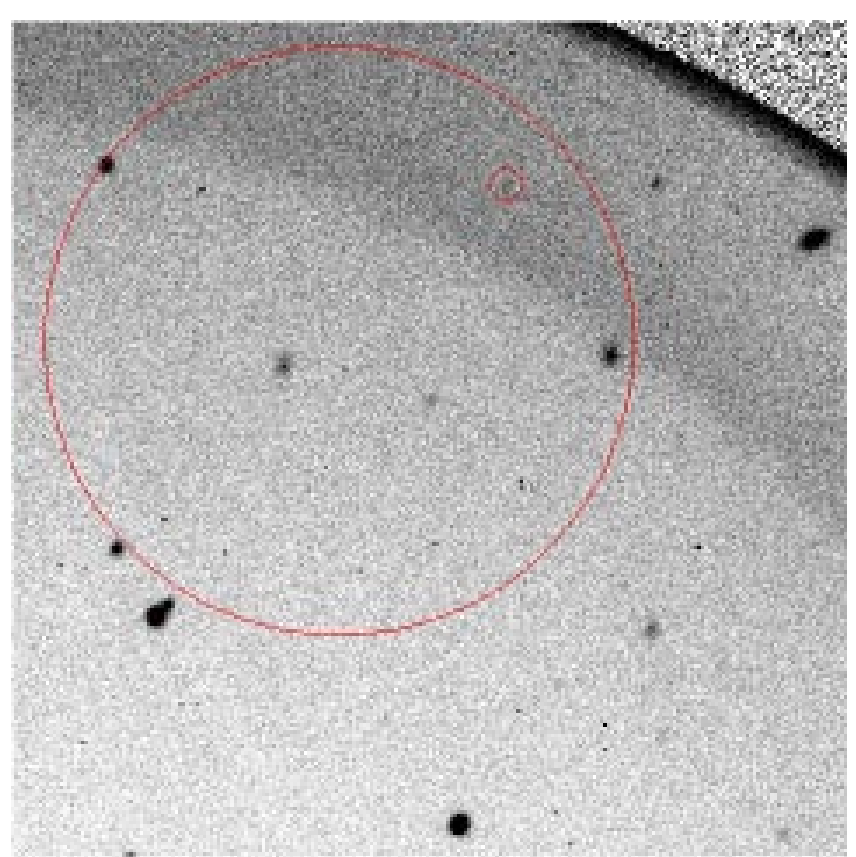

Fig. 1. Part of the $R$ frame taken at Calar Alto on Dec. 27.9. The proposed OT is located in the upper right hand region of the error circle of 1SAX J1257.3+5924 (radius: 1'.5), inside the smaller circle in the darker band. Size of the field: $4^{\prime} .24 \times 4^{\prime} .24$. The limiting magnitude is $R \sim 20.5$. North is at top, East is to the left

circle of 1SAX J1257.3+5924. Inside this circle an object was detected at $\alpha_{2000}=12^{\mathrm{h}} 57^{\mathrm{m}} 10^{\mathrm{s}}, \delta_{2000}=+59^{\circ} 24^{\prime}$ $43^{\prime \prime}$ (error box radius of $3^{\prime \prime}$, Fig. 1). It was near the border of the frame, where the instrumental background is higher; however, the reliability of the detection is better than $2 \sigma$. Other fainter stars can be clearly detected in the same part of the frame. It should also be noted that this field was imaged at very high airmass $(\sec z=4.7)$. The object was not present in two summed $R$ frames (limiting 
Table 1. Journal of the optical observations of the part of the GRB 971227 field including the OT

\begin{tabular}{ccccc}
\hline Date & Time (UT) & Telescope & Filter & Exposure (s) \\
\hline 27 Dec. 97 & 21 h 31 min & 2.2 CAHA & $R$ & 720 \\
30 Dec. 97 & 03 h 49 min & 1.5 Loiano & $R$ & 1200 \\
30 Dec. 97 & 04 h 10 min & 1.5 Loiano & $R$ & 1200 \\
30 Dec. 97 & 04 h 29 min & 1.5 Loiano & $I$ & 900 \\
04 Jan. 98 & 05 h 47 min & 2.2 CAHA & $R$ & 720 \\
\hline
\end{tabular}

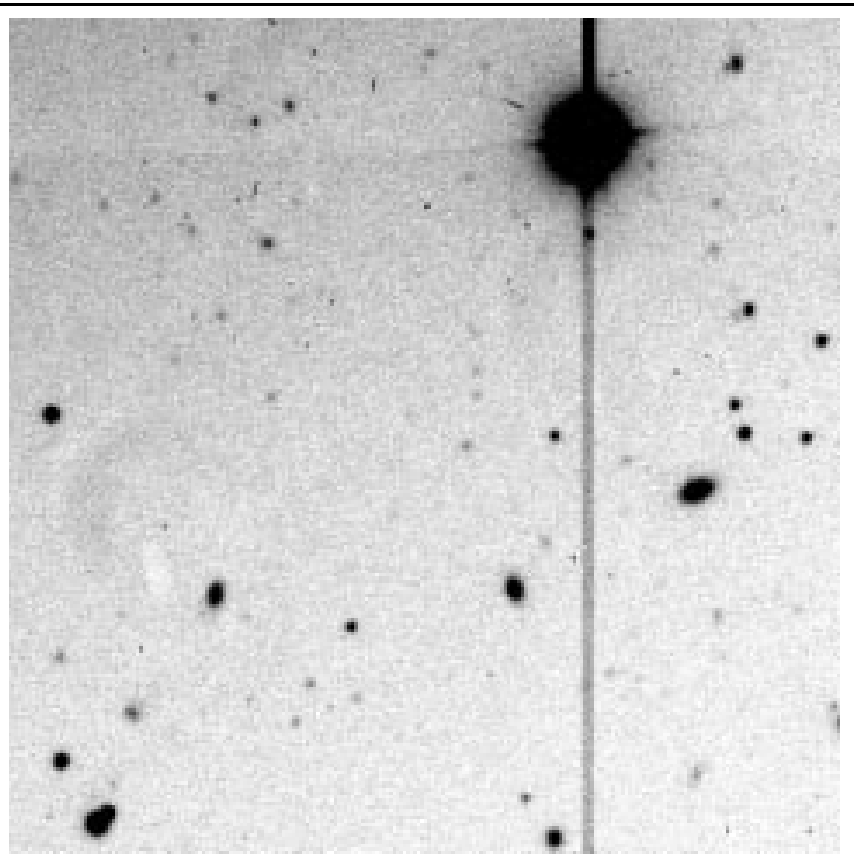

Fig. 2. Part of the two summed $R$ frames taken at Loiano on Dec. 30.16, centered on the position of the OT. Size of the field: $4.5 \times 4.5$. The limiting magnitude is $R \sim 22$. North is at top, East to the left

magnitude $\sim 22$, Fig. 2 ) and in one $I$ frame (limiting magnitude $~ 21$ ) taken by Bartolini et al. (1997) on December 30.16 UT at 1.5-m telescope of the Bologna Observatory in Loiano. Therefore it was proposed as the optical counterpart of GRB 971227 (Castro-Tirado et al. 1997; Bartolini et al. 1997).

Table 1 reports the journal of observations of the field of 1SAX J1257.3+5924 presented in this paper; some more frames not including this field have been obtained at Calar Alto and NOT telescopes. Magnitudes were calibrated referring them to the PG 0942-029 sequence (Landolt 1992). For the OT we found $R=20.0 \pm 0.3$. On December 30.8, Djorgovski et al. (1998) did not find the candidate above $R=24$ (with a limiting magnitude $R \sim 25.5$ for the frame). It was also not visible above $R=20.5$ on December 31.44 (Bond et al. 1998), and above $R=22.3$ on January 4.24 at Calar Alto.

\section{Discussion}

The OT we proposed as the counterpart of GRB 971227 is present in one frame only; therefore alternative expla- nations have to be considered. The analysis of the dark image and of the flat field allows us to exclude an instrumental defect. Moreover, the photometric profile of the OT is not compatible with a cosmic ray.

Let us consider the possible presence of an asteroid on December 27.9 in the error box of 1SAX J1257.3+5924. Down to $R=20$ there is, on average, one of such objects for $\sim 50$ square degrees at the ecliptic latitude $\left(+59^{\circ}\right)$ of the OT (Boattini 1998). Since the profile and the FWHM $\left(\sim 2^{\prime \prime}\right)$ of the OT are as the nearby stars, the upper limit of its apparent motion should be $2^{\prime \prime} / 720 \mathrm{~s}$. This constrains its position farther than the orbit of Saturn. Taking into account the dimensions of the field, the probability that the OT is an asteroid moving with a speed less than $4^{\prime}$ /day is $<10^{-7}$. The probability to find a small planet near one of its stationary points or a head-on meteorite in a field of radius 1.5 is still lower.

Thus we can find no reason to reject the object detected at Calar Alto. If this object is real and is the optical counterpart to GRB 971227, the observations taken at Loiano and by Djorgovski et al. (1998) fix severe constraints on its light curve, which shows one of the steepest declines known until now $(\alpha>1.8)$. It can be noted that among the eight so far detected OTs likely related to GRBs, also GRB 980326, GRB 980519 and (in the first part of the light curve) GRB 970228 present rapid power law decays with $\alpha>2$.

These short-lived afterglows and the steepness of their decline lead us to think of a less energetic and less extended phenomenon, which could characterize a particular class of GRBs. In some cases (GRB 970616, GRB 970815, and possibly GRB 970111) the non-detection of optical counterparts could also be explained with this kind of rapid optical decline.

Acknowledgements. We wish to thank the referee D. Lamb for his comments and suggestions, and A. Boattini for significant discussions. This work was partially supported by the University of Bologna (Funds for selected research topics), by Russian Fund of Fundamental Research, by Spanish CICYT grant ESP95-0389-C02-02 and by German Bundesministerium für Bildung, Wissenschaft, Forschung und Technologie (BMF/DLR).

\section{References}

Bartolini C., Guarnieri A., Lolli M., et al., 1997, IAU Circ. 6800

Boattini A., 1998 (private communication)

Bond H.E., Balam D., Sahu K.C., 1998, GCN 22

Castro-Tirado A.J., et al., 1997, IAU Circ. 6800

Coletta A., Gandolfi G., Smith M., et al., 1997, IAU Circ. 6796

Djorgovski S.G., Kulkarni S.R., Ramaprakash A.N., 1998, GCN 25

Landolt A.U., 1992, AJ 104, 340

Piro L., Soffitta P., Butler R.C., et al., 1997, IAU Circ. 6797 